a dignified and courteous manner. You try to convey to him the idea that you are interested in his case and will do all you can to help him. You take his family history, drop a brief word of sympathy if he has had recent deaths or congratulate him on the virility of his people if they are all alive.

As all religious societies recognize the value of music to place the minds of the congregation in a receptive mood, so the physician must recognize the hypnotic value and effect of the human voice, properly modulated, in calming his patients' nerves and getting their confidence.

Having passed through the usual careful physical examination which should always be complete as possible, considering the nature of the case, the patient is then assured that "there is hope." (That one phrase made millions for Munyon.) If the case is one in which there is no hope, it is well to inform the relatives of the patient of the facts. Whether we are justified in telling a patient is one that depends on the circumstances.

The patient who visits a physician and is told that, with care, and the following out of directions, there is every prospect of his being helped and eventually cured, will leave that office feeling better in mind and body because the suggestions have had a favorable reflective action.

If the physician dispenses his own drugs, the patient sees the medicine, hears the assuring words of the physician, is impressed with the time at which it should be taken, and feels the package when placed in his hands.

The physician has given his message to a receptive mind, and his success is assured in $80 \%$ of his cases. Why? Because the most primitive sensations of his patient have been appealed to and stimulated in a most powerful manner. His visual sensations have recorded the courteous manner of his reception and he has seen his medicine prepared. His auditory sensations have been pleased with a well-modulated voice which has assured him relief from his physical conditions, and his tactile sensations have felt the package containing the remedy which is to help him.

His physician has suggested that he will be well; he feels that the doctor is in sympathy with him, and unconsciously he tries to imitate a man of stronger mentality and fall in line with his suggestions.
By association, any previous success that the physician may have had with the patient, or a remembrance of some friend who has been successfully treated, at once tends doubly to impress and fix on the mind of the patient the thought that he will be well. Associations of this kind may be stimulated by the physician who uses tactful suggestions which leave in the mind of the patient only favorable sensations.

A large proportion of patients have mental disturbances of brief duration, as well as physical ones. Ability to treat those mental or physical conditions is greatly enhanced by a knowledge of psychology. The use of psychology at the present time by the army and by large industrial concerns in picking men of efficiency should impress us all with its impor. tance and value.

Let me repeat: as much knowledge of medicine and its allied branches as one can absorb is indispensable, but, without a working knowledge of the elements of psychology, one's years of preparation are liable to go to waste.

One thing more: the study of psychology is a review of the behavior of all peoples, at all times and under all conditions. It is a college education in itself. Why not look into it?

\section{(Original Artirleg.}

\section{RENAL AND URETERAL STONE.*}

By Benjame Tenney, M.D., F.A.C.S., Boston.

Some years ago a five-year period in the records of the Massachusetts General and of the Boston City Hospital showed that a trifle over three per cent. of the surgical admissions could be classed as urinary cases. Of these urinary cases a little over one-third, or one per cent. of the total surgical admissions, were treated for stone in the urinary tract, and the proportion may have increased with the greater perfection in urinary diagnosis which has been acquired since 1915. Certainly a lesion which may be expected at least once in every hundred surgical cases is worth keeping constantly in mind. Of 1140 cases, quickly assembled from the literature and not duplicated, 668 were in males and 472 were in females.

Many studies of the composition of urinary stones lead to the same conclusion, as stated.by Morris, that they may be classed as primary and secondary. "Primary calculi are composed

* Read before the East Boston Medical Society, October, 1917. 
of certain salts or other ingredients precipitated from the urine in the kidney independently of any preceding change in that organ and of any septic condition in the urinary passages. Microörganisms thus take no part in this formation. Secondary calculi occur in kidneys (and bladders) which have been the seat of previous disease (or injury), whereby lime salts are precipitated from the urine as a result of chemical changes caused by the presence of microörganisms."

Most calculi are built up in concentric layers around a nucleus, and these layers may consist of the different urinary salts in almost any order. An examination of the collection of stones in the Hunterian Museum led the chem. ist to consider the calculus with a urate nucleus as formed in infancy, that with a uric acid nucleus as formed in young adult life, and that with an oxalate of lime nucleus as formed in middle life.

The origin of urinary stone is not yet fully determined. The older theory is that of "diathesis," whatever that may be, whereby one person out of many, living the same life, eating and drinking alike, precipitated his urinary salts in the form of a palpable stone. Another theory requires a bacterial origin for all stones, in spite of the fact that most stones are unilateral, while bacterial excretion is bilateral. A more recent suggestion is that of partial obstruction of outflow, producing somewhere a slowing of the urinary current. The "diathesis" may finally be found to be some combination of the last two factors.

It is generally agreed that almost all urinary stones originate in the kidney, and they are found below the kidney, and often increase in size after migration. We find crystalline deposits in the kidney substance both at autopsy and on the operating-table. I distinctly remember one patient whose $x$-ray examination showed two shadows, one produced by a calculus free in the kidney pelvis, and the other by a little pocket of gritty crystals near the lower end of the kidney substance. An interesting article by Bolognesi, on "Lime Infarcts in the Kidney," describes the appearance of these deposits after the administration of various chemicals by mouth and following the injection of certain liquid antiseptics directly into the kidney. In all the instances the deposit was in or near the tubules, and not in the glomeruli. How often these and other crystalline deposits occur in our kidneys we have no means of knowing, but the proportion of men and women who at intervals feel a sudden sharp stab of pain, sometimes referred to the rectum and sometimes to the bladder or genitals, is really quite large. The sensation described differs only in duration and degree from that produced by a visible concretion.

Probably most of us have erystalline infarcts in our kidneys at some time in our lives, and most of us work them out while they are still too small to produce more than trivial symptoms. The individual who retains them in the kidney substance and increases their size, ultimately discharges them into his kidney pelvis where they probably produce the first subjective disturbance. The crystalline deposits are found in the region of the uriniferous tubules rather more than in the glomeruli, and this may have some bearing on their migration into the pelvis. Further than this we have occasional pelvic diverticula whose shape is such that a visible fragment might easily be ratained and grow therein until some accident shifts it to the upper opening of the ureter.

Of 654 cases of renal and ureteral stones, reported by Braasch from the Mayo Clinic, 56\% were removed from the kidney and the rest were passed, or removed from the ureter. At the present time we can say that a few more than half of these stones are recognized while yet in the kidney, and that this is probably due to some anatomical peculiarity which retains them there until they are too large to escape.

After they enter the ureter they may be stopped at either of the three narrowings, the first about two inches below the kidney, the second just above or below the pelvic brim, and the third and narrowest in the vesical end of the ureter. About two-thirds of the operated cases have had stones removed from the lower end of the ureter. The history of a stone in the bladder is not unlike that of a stone in the kidney pelvis; probably most which can get through the ureter pass out through the urethra, giving minor discomfort. Some pass with great pain, some are caught at one of the narrow spots, and others are retained within the bladder in a diverticulum, or because the shape of the internal meatus prevents the stone from engaging in it. That this theory rests on solid ground is shown by the fact that one patient out of every eight operated for prostatic 
obstruction, in a series of 816 cases, had vesical any of these symptoms in the past. With stone. some patients the diagnosis makes itself, and

To sum up: we do not understand the mechanism by which a erystal too large for a urinary tubule reaches the kidney pelvis,--perhaps a constant urinary pressure behind it, slight but persistent. Its retention in the pelvis may well be the result of some peculiarity in the shape of a calyx of the kidney.

Once in the ureter, we have the peristaltic action of the ureter assisted by the pressure of the urine, if the stone has entirely blocked the passage, to carry it along. Once in the bladder its further progress is determined by the presence or absence of anatomical obstruction.

Urinary stones produce quite different symptoms when obstructing the urinary outflow from those described when the stone is quiet and the urine can flow past. Urinary obstruction is far more common than any one cause for it, and the subjective symptoms of such obstructions are practically the same whatever the cause. On the other hand a "sleeping" stone may produce so few and such indefinite symptoms that its discovery by $x$-ray or in the course of some abdominal operative work may be quite unexpected. For these reasons it is impossible to give any set of symptoms which will allow us to make a diagnosis from the case history and ordinary observation.

Usually during an attack of renal colic the quantity of urine is diminished, while the frequency of urination is increased, and this is a valuable indication in cases with acute and severe symptoms. Complete anuria is more commonly associated with urinary stone than with any other cause. The urine passed by a man or catheterized from a woman will often give valuable evidence for or against urinary stone if the sediment be promptly examined under the microscope. Obvious blood in the urine helps in the diagnosis, but the presence or absence of albumen counts for little.

The group of symptoms characteristic of urinary stone includes hematuria, subcostal or vesical pain, and unusual frequency in urinating during activity. This combination never exists without some serious lesion in the urinary tract, though the lesion is not always a stone. The converse is also true-that a stone may be lying in the kidney, the ureter, or the bladder without any symptom of this group showing for a long period, and our history-taking may fail to reveal the presence of with others it is made only after disappointments at the failure of varied surgical adventures to give relief.

The $\mathrm{x}$-ray has given the most reliable assistance in diagnosis of all the methods used, but the 10 or $15 \%$ of error in the hands of most competent operators forbids our complete trust in its showing. The cystoscope and ureteral catheter are helpful in making the diagnosis and sometimes give a positive answer, but more often they contribute only a piece of evidence to be weighed with the rest. Fortunately, with these aids and a careful history, a definite and accurate diagnosis can be made in the majority of cases.

If it is pain that brings the paiient and physician together, the patient's hand will locate the pain more definitely than his words. If he locates it about the twelfth rib, think of obstruction to the urinary current on that side. This obstruction may be stone, or clot of blood or pus, or kink or stricture in the ureter. The pain may be anything from a dull aching in the upright position only, to a prostrating agony with vomiting, cold perspiration, and rigid abdominal muscles. It may simulate an appendix or gall-bladder colic, an acute intestinal obstruction or perforation, or the rupture of an extra-uterine pregnancy.

Ureteral colic reaches its maximum of pain rather suddenly, and is peristaltic in character, corresponding to the ureteral contractions which are more frequent than those of the intestine, but the ache of a distended kidney is more likely to be a steady grind, and to be relieved by horizontal posture.

If hematuria is the symptom which occasions the professional visit, we can confine our study to the urinary tract and inquire into the details of its appearance before, with, or after urination, its relation to activity and posture, and its association with pain and functional disturbance. In these cases the cystoscope is indispensable as well as the best means of destroying bleeding papillomata.

If the female patient comes for relief from frequent urination, urgent urination, leakage, or pelvic discomfort, the physician should always keep in mind the possibility that a ureter or vesical stone may be the cause, even if there be some genital injury or abnormality. Here again the cystoscope and $\mathrm{x}$-ray, separately or 
combined for pyelography, will give invaluable assistance in determining the cause of the symptoms.

Fortunately, urinary stone is not rapidly fatal. Death from calculus anuria seldom occurs before the fifth day, and the other fatalities from urinary stone are much slower. Ureteral and renal colics quickly reach their maximum of pain and general disturbance while the other acute processes with which they may be confused do not reach their maximum of general disturbance so rapidly.

The mistakes of diagnosis in the atypical cases are due to various things. If a man takes his responsibility to a specialty more seriously than he does his general pathology he will include too many outside cases in his list honestly, because his questions are unconsciously framed to elicit certain answers. Another source of error is the persistence in our minds of names that mean nothing definite, such as lumbago, neuralgia, inflammation of this and that unseen organ, and the like. Another is the eagerness to give relief by mechanical means with which the practitioner is familiar, and which have brought him honor in cases which justified his quick diagnosis.

It is a melancholy fact that most of us will die before we attain to perfection in diagnosis or anything else, but careful history-taking and the equally careful use of the diagnostic methods available in a large town will be followed by fewer mistaken diagnoses in the urinary tract than in almost any other human department.

\section{TREATMEN'T.}

There is but one,-removal either by direct operation or through the natural passages. Attempts at solution or disintegration of a real calculus by drugs or waters are followed by the same degree of success that obtains without their use. Sixty-four out of 654 cases of renal and ureteral calculi reported by Braasch were eliminated without a cutting operation. Many patients are so little incommoded by their stones that they prefer to take the chance of elimination rather than have prompt surgical relief, and for them we can try distention of the bladder to the limit of tolerance, rectal irrigation with hot water, or the various fluids suggested for use through the ureteral catheter. Operating cystoscopes fitted with scissors for slitting the ureter orifice, and forceps for

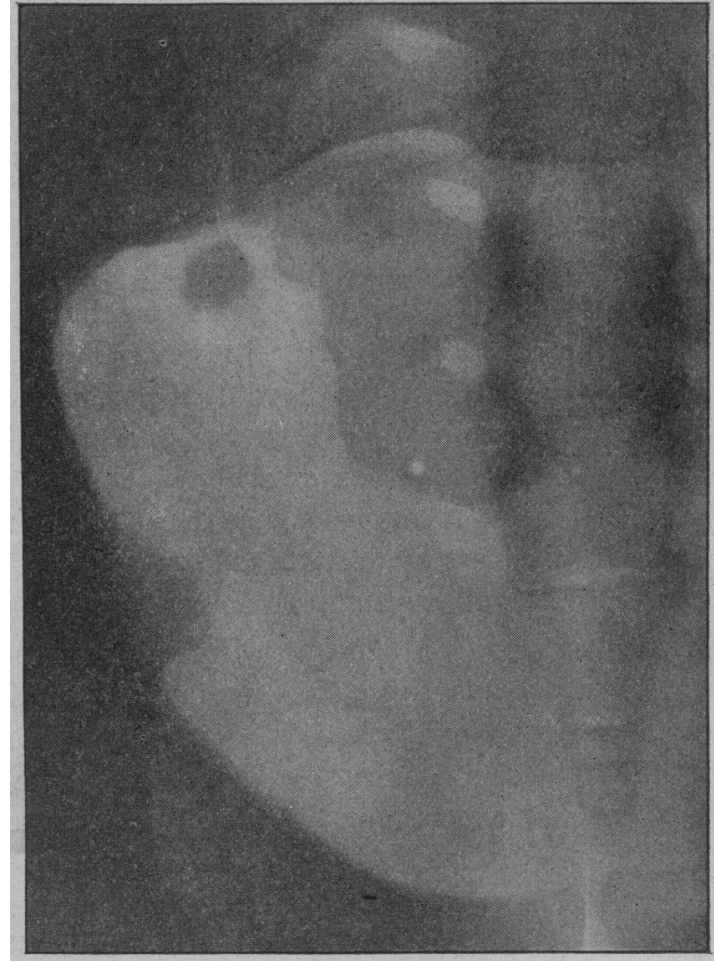

Fig. 1. Lreteral. STune. May, 1013. E. W. S. (Kindness of Massachusetts Homeopathic Hosnital, X-ray Dept.) grasping the stone, have been used with success by some urologists.

For the control of pain during a colic, opium in the form of Dover's powder or morphine hypodermically are best. Inhalation anesthesia is preferred by some because it can be discontinued at once when the pain stops. Prompt relief can be obtained by open operation on any part of the urinary tract, with very low mortality. Posterior pyelotomy is preferred for renal stone when the kidney can be well mobilized. Nephrotomy is often necessary because of adhesions or a short pedicle, and nephrectomy can be done if the kidney be damaged beyond comfortable repair and its partner be known to be competent. Suture is necessary after nephrotomy or pyelotomy.

Stones in the ureter are best removed by cutting directly down on them. Suture is desirable and usually successful, but a fistula from a ureter usually closes. Removal of these stones is in one way like the removal of gallstones,- - the anatomical conditions which accompanied their formation still exist and may favor re-formation or themselves produce symptoms. For this reason the suggestion of Fowler that after extraction of a renal stone the kidney should be fixed parallel to the twelfth 


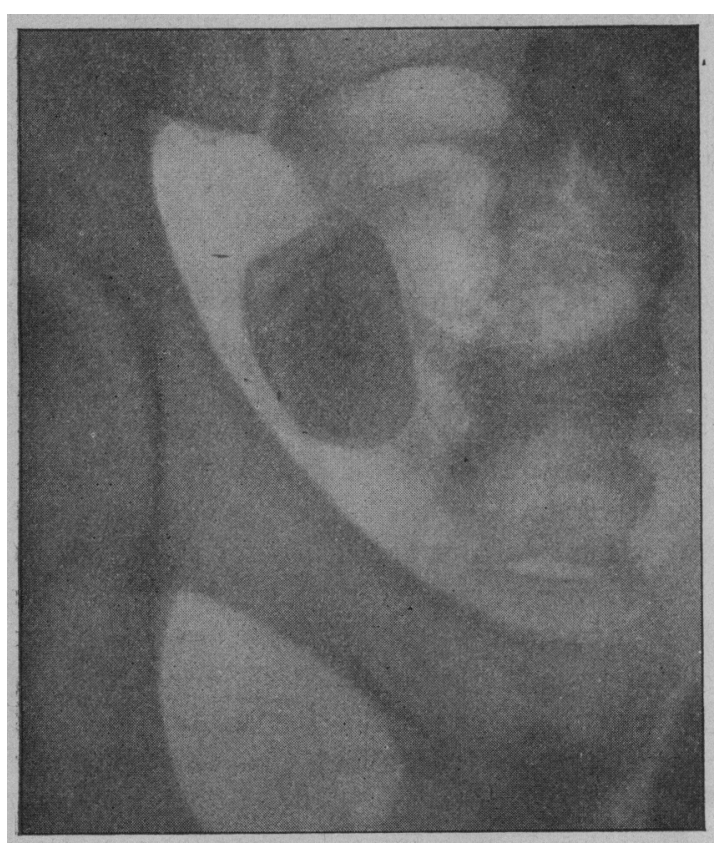

Fig. 2. Ureteral Stone. March, 1916. F. W. S. Taken by Dr. A. W. George.

rib is worth considering. Such a position might make the difference between the retention and growth of a crystal in a calyx and its prompt discharge through the ureter. After removal of ureteral stones occasional dilatation of the ureter is advisable and sometimes very necessary.

Without operative treatment the stone may be expelled, a $10 \%$ chance, according to the figures quoted, and certainly a painful experience. If the stone is allowed to stay in the kidney it will cause some degree of discomfort and disability and some darnage to the kidney structure if too large to engage in the ureter. If the stone remains in the ureter, the discomfort and disability may be greater, with the probability of producing a hydronephrosis, and the possibility of a periureteral abscess from perforation of the ureter, or fatal anuria. Another possibility is shown in the $\mathrm{x}$-ray plates accompanying this paper. The first plate was taken in 1913 and the second in 1916. The stone lay in a dilated ureter, adherent to the walls, and was easily crushed during extraction.

Some of my cases are briefly reported which show some special feature of interest.

Case 1. Referred by Dr. John L. Ames.Three years previous to my examination she had spent three days in bed with severe pain in the lower abdomen, vomiting, and frequent urination. At the time of her examination her urination was $\frac{4-6}{0}$. Catheter urine was perfectly clear and albumen-free. She had large uterus with multiple fibroids. At the time of hysterectomy a calculus was felt and removed from the left ureter, but at no time could any further history, suggesting ureteral calculus, be obtained.

CASE 2. Referred by. Dr. F. A. Bardwell.Nine years before, his appendix was removed. Four years later he was operated for acute intestinal obstruction and had a fecal fistula lasting two years. One week before his visit he had severe pain in right back, lasting six hours. This has recurred and prevented sleep. $\mathrm{He}$ had never had pain of this sort before, and no nausea with the pain, no radiation of pain, and to pain on left side. His right kidney was tender and also the line of right ureter. Urine showed pus and blood. Cystoscopy found hypertrophy of the ureteral bands. Both catheters passed easily and delivered cloudy urine from the painless side. An x-ray plate later explained this by showing a "sleeping" stone in the left kidney and a calculus in the right ureter. Both were easily removed at the same operation.

CASE 3. Referred by Dr. George Bowles.Frequent and imperative urination, with leakage for years. Pain and tenderness on right side, contracted bladder and difficult cystoscopy. X-ray showed shadow in the region of left kidney. This was operated first under the idea of "crossed pain," which was then being discussed. A calcified lymph node was removed from the region of a normal-appearing kidney, and later a tubercular right kidney was removed, relieving the patient of her symptoms.

CASE 4. Referred by Dr. A. D. MacLennan.Frequent urination for five years; two attacks of colicky pain on the left side, with increased frequency of urination, and one on the right side. Hematuria once. No tenderness over either kidney; urine contained pus and blood; cystoscopy showed a normal bladder, with tender left ureter and blood in both catheter specimens. X-ray showed no shadow in either kidney. I did not see him again, but learned that his left kidney was removed at the Boston 
City Hospital on account of calculus pyonephrosis about a year later.

Case 5. Referred by Dr. Harry W. Goodall.-Hematuria for three weeks, with slight pain in lower left side for a few days. Blood always mixed with urine. No pain at any part of urination. No night urines. No pain on either side. Cystoscopy negative and no gross blood in either catheter urine; some microscopic in both, more in right. Three months later had attack of severe pain on right side, with tenderness over kidney and along right ureter. $\mathrm{X}$-ray showed stone in right side, probably in upper ureter. This was removed without diffculty.

CASE 6. Referred by Dr. Walter H. Young. -Pain in left side in periods lasting from three to nine hours, vomiting, urination hourly by day, two or three times at night. Urine contained pus and blood. Cystoscopy negative, purulent urine from left ureter. $X$-ray positive. At operation a double ureter was found with the stone easily removed from the second.

CASE 7. Previously published. Attacks of colic for two years, pain extending to bladder. Attacks came on gradually, with diminution of urine, followed by an hour of increasing discomfort up to the colic. Much urine after pain stopped. Patient was very thin, and a very tender mass, freely movable, could be felt above the pelvic brim. A stone was easily removed from the ureter.

Case 8. Referred by Dr. Samuel Breck.Case of calculus in both ureters, with pain sometimes on the left side and sometimes on the right, with the usual frequency, and purulent urine. X-ray showed shadows on left; and operation found and removed stone from upper end of ureter. Probe and catheter failed to find other concretions on this side. Convalescence was disturbed by severe pain in the right side, and vaginal examination found a tender nodule at lower end of right ureter. Oil was injected into the right ureter without immediate relief. Patient was transferred to a contagious hospital with scarlet fever and passed her right concretion there.

In two other cases, one seen with Dr. Henry F. Dearborn of Lawrence, and the other the wife of a physician, it has been possible to reach a tender nodule by vagina and identify it with the ureter. The nodule in these cases has been several times the size of its enclosed calculus and mostly composed of fat firmly adherent to the outside of the ureter. That the presence of such a tender nodule does not settle the diagnosis is shown by a patient referred by Dr. Daniel F. Murphy of Beverly.

She had a tender left nodule in close relation to the left ureter, urinary symptoms, left-sided pain, a positive shadow on successive $\mathrm{x}$-ray plates, and a previous laparotomy. The only negative finding was the absence of obstruction in passing a ureteral catheter. At operation the ureter appeared normal, but was opened and explored its full length with a flexible metal probe without finding stone or other obstruction. In front of the ureter was an enlarged lymph node which probably had enough lime in it to produce the x-ray shadow.

Another case with positive $\mathrm{x}$-ray and negative findings was referred by Dr. C. O. Kepler. In this patient, a young woman, there was no palpable nodule and nothing was discovered at the operation to account for the shadow.

To sum up: urinary stone produces about $1 \%$ of surgical cases and should, therefore, always be considered among the causes of loin and pelvic pain. A small stone may be passed through the natural tubes-about a $10 \%$ chance-and its passage may be assisted in various mechanical ways, but we have no reliable evidence of the solution of any such concretion. Nature's method may cause great and repeated physical distress, with danger to the kidney, and should not be endured beyond a reasonable time.

The surgical removal of stones from the kidney or ureter is safely possible in most cases, and desirable for all who have demonstrated their inability to pass them after such assistance as can be given through the cystoscope.

MOBILIZATION OF THE KNEE· JOINT IN SUSPENSION - TRACTION TREATMENT OF FRACTURE OF THE FEMORAL SHAFT.

BY G. A. Moore, M.D., Brockton, Mass.

Soon after the outbreak of the present war it became evident to most military surgeons that the generally accepted methods of treating fractures of the shaft of the femur in civil practice 